\title{
Amphiphilic Polymer-Modified Uniform CuFeSe 2 Nanoparticles for CT/MR Dual-Modal Imaging
}

\author{
Min Wu, ${ }^{1,2}$ Shaozhi Fu $\left(\mathbb{D},{ }^{3}\right.$ Jian Shu $\mathbb{D}^{1},{ }^{1}$ and Kequan $\mathrm{Yu}^{4}$ \\ ${ }^{1}$ Department of Radiology, The Affiliated Hospital of Southwest Medical University, Luzhou, Sichuan, China \\ ${ }^{2}$ Department of Radiology, People's Hospital of Chongqing Yubei District, Yubei District, Chongqing, China \\ ${ }^{3}$ Department of Oncology, The Affiliated Hospital of Southwest Medical University, Luzhou, Sichuan, China \\ ${ }^{4}$ Department of Surgery, The First Affiliated Hospital of Chongqing Medical University, Chongqing, China
}

Correspondence should be addressed to Shaozhi Fu; shaozhifu513@163.com and Jian Shu; shujiannc@163.com

Received 19 April 2020; Revised 6 December 2020; Accepted 20 December 2020; Published 30 December 2020

Academic Editor: Changning Wang

Copyright (c) $2020 \mathrm{Min}$ Wu et al. This is an open access article distributed under the Creative Commons Attribution License, which permits unrestricted use, distribution, and reproduction in any medium, provided the original work is properly cited.

\begin{abstract}
Recently, magnetic photothermal nanomaterials have attracted much attention in the diagnosis and treatment of cancer. In this study, we developed the ultrasmall magnetic $\mathrm{CuFeSe}_{2}$ nanoparticles for CT/MR dual-modal imaging. By controlling the reaction time and condition, $\mathrm{CuFeSe}_{2}$ nanoparticles were synthesized by a simple directly aqueous method. After modification with copolymer methoxy polyethylene glycol-polycaprolactone (MPEG-PCL), the obtained MPEG-PCL@CuFeSe ${ }_{2}$ nanoparticles showed excellent water solubility, colloidal stability, and biocompatibility. In addition, they also exhibited superparamagnetism and X-ray's characteristics. For these properties, they will become ideal nanomaterials for CT/MR dual-modal imaging.
\end{abstract}

\section{Introduction}

In recent years, nanotechnology has been widely used in the field of biomedicine, such as the development of tumor therapeutic drugs and molecular imaging probes [1, 2]. Among them, semiconductor nanomaterials not only have strong fluorescence characteristics but also have fine and uniform particle size, while carbon dots and organic polymer fluorescent nanomaterials have low biological toxicity and relatively stable chemical properties $[3,4]$. Many fluorescent nanomaterials, for example, gold-based nanomaterials $[5,6]$, carbon-based nanomaterials [7], conjugated polymeric nanomaterials [8], and graphene [9-11], are extensively used in photoacoustic imaging and photothermal therapy of tumors, which achieve the purpose of the integration of diagnosis and treatment of tumors.

$\mathrm{CuFeSe}_{2}$ is classified as I-III-VI ${ }_{2}$ ternary chalcogenide semiconductor materials, which has interesting optical, electronic, and magnetic properties. Until now, many studies about $\mathrm{CuFeSe}_{2}$ mainly focus on the synthesis methods as well as magnetic and optoelectronic properties [12-16]. There are few reports about its application in diagnosis and cancer treatment
$[17,18]$. Therefore, we will try to explore the potential value of its application in the field of imaging diagnosis.

Currently, the $\mathrm{CuFeSe}_{2}$ nanostructures can be prepared by the solvothermal reaction [19] and the high-temperature solid phase reaction [16]. The products which are often synthesized tend to have a nonuniform size and are prone to agglomeration. Therefore, in an effort to overcome their disadvantages of $\mathrm{CuFeSe}_{2}$ nanostructures, we attempted to prepare with biodegradable copolymer loaded $\mathrm{CuFeSe}_{2}$ nanocrystals to increase the solubility in aqueous media.

MPEG-PCL is an amphiphilic copolymer, and many studies demonstrate that MPEG-PCL copolymer can significantly improve water solubility of hydrophobic drugs and keep better stability [20-22]. Therefore, it can be used to load $\mathrm{CuFeSe}_{2}$ nanoparticles for molecular imaging in vivo. First, the properties of PCL, such as crystallinity, tensile strength, and hydrophobicity, can be easily modulated, and thus, the loading capacity for hydrophobic $\mathrm{CuFeSe}_{2}$ nanoparticles can be tuned. Second, PEG is nonimmunogenic and highly hydrophilic [23]. Surface coating with PEG can prolong nanoparticle circulation time in vivo, leading to better-enhanced imaging results. 
Every imaging modality has its strengths and weaknesses [24]. For instance, X-ray computed tomography (CT) owns its advantages, such as fast acquisition time, large tissue penetration depth, and high spatial resolution, but it has a poor soft-tissue contrast. Magnetic resonance (MR) imaging possesses favorable spatial and soft-tissue resolution, and it can implement multisequence, multiparameter imaging, but its limitation is low sensitivity. Nuclear imaging techniques, including single photon emission computed tomography (SPECT) and positron emission tomography (PET), exhibit high sensitivity and are quantitative, but along with a poor spatial resolution. However, multimodal imaging can improve the accuracy of cancer diagnosis by combining two or more imaging modalities into one system $[25,26]$. It overcomes the intrinsic limitations of single modality.

In this study, the ultrasmall magnetic $\mathrm{CuFeSe}_{2}$ nanostructures were prepared by a simple direct aqueous method. After modification with methoxy polyethylene glycol-polycaprolactone (MPEG-PCL), the biosafety of obtained MPEG-PCL@CuFeSe ${ }_{2}$ nanoparticles was evaluated. Lastly, the X-ray attenuation property and $\mathrm{T}_{2} \mathrm{MR}$ relaxometry of MPEG-PCL@CuFeSe $\mathrm{CPs}_{2}$ in vitro/in vivo were measured to explore the potential application of these NPs as dual-modal CT/MR imaging contrast agents.

\section{Experimental Section}

2.1. Materials. Copper (II) chloride dihydrate $\left(\mathrm{CuCl}_{2} \cdot 2 \mathrm{H}_{2} \mathrm{O}\right.$, $\geq 99 \%$ ), ferrous (II) sulfate heptahydrate $\left(\mathrm{FeSO}_{4} \cdot 7 \mathrm{H}_{2} \mathrm{O}\right.$, $\geq 99 \%$ ), selenium powder ( $\mathrm{Se}, \geq 99.5 \%$ ), sodium borohydride $\left(\mathrm{NaBH}_{4}, 99 \%\right)$, L-cysteine (cys, $\left.\geq 99 \%\right), \mathcal{E}$-caprolactone ( $\varepsilon$-CL, Alfa Aesar, USA), poly(ethylene glycol) methyl ether (MPEG, Mn = 2000, Aldrich, USA), and stannous octoate $\left(\mathrm{Sn}(\mathrm{Oct})_{2}\right)$ were bought from Sigma-Aldrich (USA).

2.2. Synthesis of CuFeSe ${ }_{2}$ Nanoparticles. For the synthesis of $\mathrm{CuFeSe}_{2}$ nanoparticles, $78.96 \mathrm{mg}$ of Se powder was dispersed in $100 \mathrm{~mL}$ of Milli-Q water, and then $75.60 \mathrm{mg}$ of $\mathrm{NaBH}_{4}$ was added to reduce it at ambient conditions with protection of nitrogen flow for one hour. After Se powder was completely reduced, a colorless solution is obtained. A $10 \mathrm{~mL}$ mixture of $\mathrm{CuCl}_{2} \quad 2 \mathrm{H}_{2} \mathrm{O}$ (85.24 mg), $\mathrm{FeSO}_{4} \quad 7 \mathrm{H}_{2} \mathrm{O}$ (139.01 mg), and L-cysteine $(121.20 \mathrm{mg}$ ) was separately prepared, and then the above mixture was added into the selenium precursor solution immediately to form a black solution. The resultant solution was collected after centrifugation with a speed of $3500 \mathrm{rpm}$ for twenty minutes to remove impurities. The purified $\mathrm{CuFeSe}_{2}$ solution was stored at $4^{\circ} \mathrm{C}$ for further characterization and application.

2.3. Functionalization of $\mathrm{CuFeSe}_{2}$ Nanoparticles. Methoxy poly (ethylene glycol)-poly ( $\varepsilon$-caprolactone) (MPEG-PCL) used in this study was synthesized by ring-opening polymerization of $\varepsilon$-CL on MPEG using $\mathrm{Sn}(\mathrm{Oct})_{2}$ as catalyst, according to a previous report [27]. The MPEG-PCL colloidal solution was prepared by liquid rotary evaporation method.

For functionalization of $\mathrm{CuFeSe}_{2}$ nanoparticles, the above purified $\mathrm{CuFeSe}_{2}$ solution was slowly added into the MPEG-PCL colloidal solution under ultrasonication for 4 hours, and then the MPEG-PCL-modified $\mathrm{CuFeSe}_{2}$ nanoparticles were obtained after centrifugation to remove excess and large impurities. The purified MPEG-PCL@CuFeSe solution was stored at $4^{\circ} \mathrm{C}$ for future experiments.

2.4. Characterization. The hydrodynamic diameters and zeta potentials of prepared $\mathrm{CuFeSe}_{2}$ solution and MPEG-PCL@ $\mathrm{CuFeSe}_{2}$ solution were measured by dynamic light scattering (DLS, NanoBrook 90Plus Zeta, Brookhaven, USA) at $25^{\circ} \mathrm{C}$. The size and morphology of prepared $\mathrm{CuFeSe}_{2}$ nanoparticles and MPEG-PCL@CuFeSe ${ }_{2}$ nanoparticles were characterized with a transmission electron microscope (TEM, Tecnai G2 F20, USA). The crystallography structures of $\mathrm{CuFeSe}_{2}$ nanoparticles and MPEG-PCL@CuFeSe $\mathrm{C}_{2}$ nanoparticles were characterized by using an X-ray diffractometer equipped with $\mathrm{Cu} \mathrm{K} \alpha$ radiation $(\lambda=0.15406 \mathrm{~nm})$. A scanning rate of $0.1^{\circ} / \mathrm{s}$ was applied to record the pattern in the $2 \theta$ range of $10-90^{\circ}$. The T2-weighted images of MPEG-PCL@CuFeSe $e_{2}$ at different concentrations were scanned under a 3T clinical MRI scanner at room temperature. After the T2-weighted MR images were acquired, the signal intensity was measured by a manually drawn region-of-interest for each sample.

2.5. Cell Culture and Cytotoxicity Assessment. 4T1 murine breast cancer cells, A549 human lung adenocarcinoma, and human normal liver cells were cultured in standard cell media supplemented with 10\% fetal bovine serum (FBS) and antibiotics $(100 \mathrm{U} / \mathrm{mL}$ penicillin and $100 \mu \mathrm{g} / \mathrm{mL}$ streptomycin) at $37^{\circ} \mathrm{C}$ in an atmosphere of $5 \% \mathrm{CO}_{2}$. All cell culturerelated reagents were purchased from HyClone (USA). The cytotoxicity of MPEG-PCL-CuFeSe ${ }_{2}$ NPs was evaluated by the MTT assay. The cells were first seeded into 96-well plates $\left(1 \times 10^{4}\right.$ cells per well) and cultured for $24 \mathrm{~h}$ and then added into different concentrations of MPEG-PCL-CuFeSe $e_{2}$ and continued to culture for $24 \mathrm{~h}$. After this, $10 \mu \mathrm{L} \mathrm{MTT}(5 \mathrm{mg} /$ $\mathrm{mL}$ ) was added. Four hours later, the supernatant medium was removed, and $150 \mu \mathrm{L}$ DMSO was added into each well to dissolve the resulting formazan crystals. The absorbance was measured at $490 \mathrm{~nm}$ using a spectrophotometric microplate reader (iMark, MA, USA). The cytotoxicity was calculated as the percentage of cell viability.

2.6. Animal Model. We acquired female BALB/c mice (6-8 weeks of age, 25-30 g of weight) from Chongqing Tengxin Biotechnology Co. Ltd. (Chongqing, China). To generate the $4 \mathrm{~T} 1$ tumor murine model, $1 \times 10^{6}$ cells in the $100 \mu \mathrm{L}$ serum-free RMPI-1640 medium were subcutaneously injected into in the right side thigh root of each mouse. All mice were selected for imaging experiments when their tumors grew to $80 \mathrm{~mm}^{3}$. 
2.7. In Vitro CT/MR Dual-Modality Imaging. Various concentrations of MPEG-PCL@CuFeSe 2 solution were dispensed in $5.0 \mathrm{~mL}$ Eppendorf tubes for CT and MR contrast imaging. The MR imaging for in vitro study was performed on a $3.0 \mathrm{~T}$ clinical magnetic resonance (MR) scanner (PHILIPS, Holland). The representative imaging parameters of the T2-weighted images were as follows: repetition time $(\mathrm{TR})=5348 \mathrm{~ms}$, echo time $(\mathrm{TE})=70 \mathrm{~ms}$, slice thickness $=1.5 \mathrm{~mm}$, slice spacing $=0.15 \mathrm{~mm}$, matrix $=256 \times 256$ pixels, field of view $(F O V)=30 \mathrm{~cm} \times 60 \mathrm{~cm} \times 25 \mathrm{~cm}$, $\mathrm{NSA}=4$, and flip angle $=10^{\circ}$. The region-of-interest was selected by drawing manually to measure the signal intensity of MPEG-PCL@CuFeSe 2 solution from the T2-weighted MR images. The CT data were acquired using a clinical CT imaging scanner (GE, USA), and X-ray attenuation values for all samples were finally calculated in Hounsfield units (HU) by averaging over the region-of-interest (ROI). CT imaging parameters were as follows: tube current $=600 \mathrm{~mA}$, tube voltage $=100 \mathrm{kV}$, and slice thickness $=0.625 \mathrm{~mm}$.

2.8. In Vivo CT/MR Dual-Modality Imaging. The $4 \mathrm{~T} 1$ tumorbearing mice were acquired before and after intratumorally (i.t.) injected with MPEG-PCL@CuFeSe $2(250 \mu \mathrm{L}, 2 \mathrm{mg} /$ $\mathrm{mL}$ ) and imaged with a $3.0 \mathrm{~T}$ clinical magnetic resonance (MR) scanner (PHILIPS, Holland) equipped with a small animal coil. The representative imaging parameters of the T2-weighted images were as follows: repetition time $(\mathrm{TR})=$ $5348 \mathrm{~ms}$, echo time $(\mathrm{TE})=70 \mathrm{~ms}$, slice thickness $=1.5 \mathrm{~mm}$, slice spacing $=0.15 \mathrm{~mm}$, matrix $=256 \times 256$ pixels, field of view $(F O V)=30 \mathrm{~cm} \times 60 \mathrm{~cm} \times 25 \mathrm{~cm}, \quad N S A=4$, and flip angle $=10^{\circ}$. The region-of-interest in the tumor area of each mouse was selected by drawing manually to measure the signal intensity of tumors from the T2-weighted MR images. The CT images were acquired before and after intratumorally (i.t.) injected with MPEG-PCL@CuFeSe $(250 \mu \mathrm{L}, 2 \mathrm{mg} / \mathrm{mL})$ on a clinical CT imaging scanner (GE, USA), and the CT imaging parameters were as follows: tube current $=600 \mathrm{~mA}$, tube voltage $=100 \mathrm{kV}$, and slice thickness $=0.625 \mathrm{~mm}$. The region-of-interest in the tumor area of each mouse was selected by drawing manually to measure the CT value of tumors from the CT images.

2.9. In Vivo Toxicity Study. The major organs/tissues were taken from mice after intravenous injection of MPEG-PCL@ $\mathrm{CuFeSe}_{2}$ (a dose of $20 \mathrm{mg} / \mathrm{kg}$ ) at 1 day, 3 days, 7 days, and 15 days postinjection, while other mice without injection were used as the control group (four mice per group). Then, the obtained major organs/tissues were fixed in $4 \%$ formalin, paraffin-embedded, sectioned, and stained with hematoxylin \& eosin (H\&E) and then imaged by using a digital microscope to evaluate the histological changes.

\section{Results and Discussion}

3.1. Synthesis and Characterizations of MPEG-PCL@CuFeSe Nanoparticles. In our experiments, MPEG-PCL@CuFeSe nanoparticles with uniform sizes and morphologies were synthesized by a simple direct aqueous method. The resultant nanoparticles were characterized by transmission electron microscopy (TEM) to determine their size and morphology. Small spherical particles with a size of $4.2 \pm 0.7 \mathrm{~nm}$ are clearly observed (Figures 1(a), 1(c)). The crystal structure of $\mathrm{CuFeSe}_{2}$ nanoparticles was observed by their high-resolution TEM (HR-TEM) image, which clearly shows the lattice fringes with an interplanar spacing of $0.325 \mathrm{~nm}$ (Figure 1(b)). The FTIR spectra showed a typical variation peak of $\mathrm{C}=\mathrm{O}$ at $1727.60 \mathrm{~cm}^{-1}$ and typical variation peaks of $\mathrm{C}-\mathrm{H}$ at $2891.05 \mathrm{~cm}^{-1}$ and $2949.12 \mathrm{~cm}^{-1}$ in the MPEG-PCL@CuFeSe ${ }_{2}$ nanoparticles, verifying the successful modification of MPEG-PCL (Figure 2(a)).

The X-ray powder diffraction (XRD) results show that both $\mathrm{CuFeSe}_{2}$ and MPEG-PCL-CuFeSe ${ }_{2}$ nanoparticles have crystal face peaks at (112) and (220), indicating that the nanoparticles are cubic crystal structures (Figure 2(b)). The content of MPEG-PCL polymer on the surfaces of $\mathrm{CuFeSe}_{2}$ nanoparticles was determined by thermogravimetric analysis (TGA) to be approximately $40.0 \mathrm{wt}$. \% (Figure 2(c)), and the TGA results demonstrate the successful coating of $\mathrm{CuFeSe}_{2}$ nanoparticles with MPEG-PCL. For the magnetic properties of $\mathrm{CuFeSe}_{2}$ and MPEG-PCL@CuFeSe $e_{2}$ nanoparticles, the superparamagnetic properties of $\mathrm{CuFeSe}_{2}$ and MPEG-PCL@CuFeSe $\mathrm{Se}_{2}$ were illustrated by the absence of a hysteresis loop in the field-dependent magnetization measurement (Figure 2(d)). According to the results from DLS, the hydrodynamic diameters of MPEG-PCL NPs and MPEG-PCL@CuFeSe2 NPs were 25.31 $\pm 2.07 \mathrm{~nm}$ and $120.91 \pm 3.44 \mathrm{~nm}$, respectively. MPEG-PCL NPs had a negative surface charge of $-21.03 \pm 1.53 \mathrm{mV}$, and MPEG-PCL@CuFeSe2 NPs had a negative surface charge of $-10.85 \pm 2.59 \mathrm{mV}$ (Table 1).

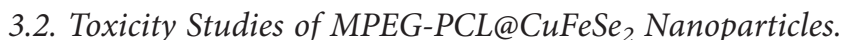
Good biosecurity is an important criterion for measuring whether nanomaterials can be applied to living organisms. Thus, in vitro MTT assay and in vivo pathological assay were investigated to test the cytotoxicity and biosecurity of MPEG-PCL@CuFeSe ${ }_{2}$ NPs, respectively. The results of MTT assay showed that the viability of the above three kinds of cells still kept above $90 \%$ in the concentration range of $0-150 \mu \mathrm{g} / \mathrm{mL}$ for MPEG-PCL@CuFeSe $e_{2}$ nanoparticles after cultured $24 \mathrm{~h}$, demonstrating the cytotoxicity is not obvious (Figure 3(a)). In order to test toxicity study of MPEG-PCL@CuFeSe ${ }_{2}$ NPs in vivo, histological assessment of tissues was performed to determine whether MPEG-PCL@-CuFeSe $\mathrm{NPs}_{2}$ caused damage to important organs. The above representative organs including heart, liver, spleen, lung, and kidney had no apparent histopathological abnormalities or lesions, compared with those of the control group throughout the entire study. Therefore, the results of in vivo toxicity indicated the good biocompatibility of MPEG-PCL@CuFeSe 2 NPs (Figure 3(b)).

3.3. In Vitro CT/MR Dual-Modality Imaging. We successfully performed in vitro CT/MR imaging experiment, and it demonstrates the potential of MPEG-PCL@CuFeSe $\mathrm{NPs}_{2}$ in 


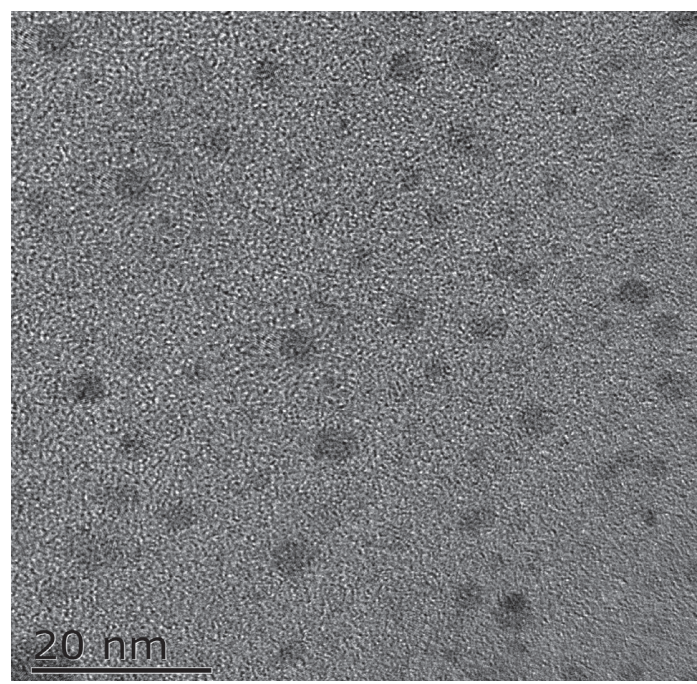

(a)

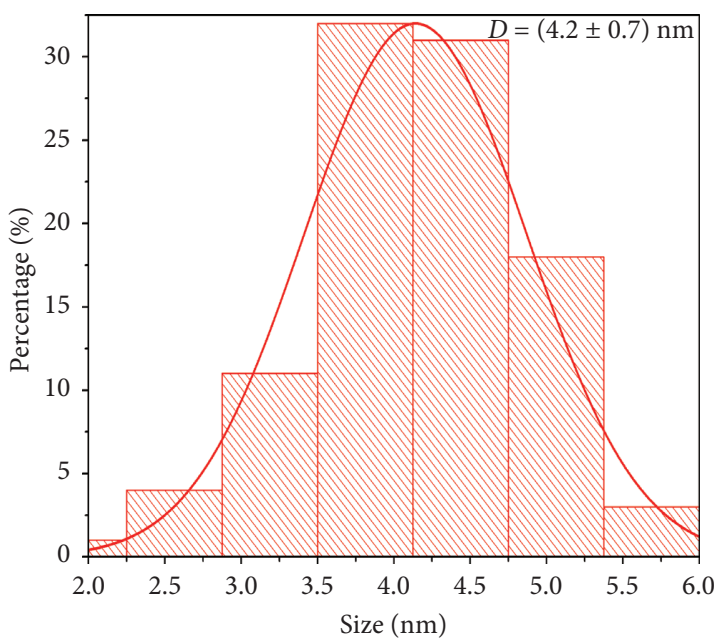

(c)

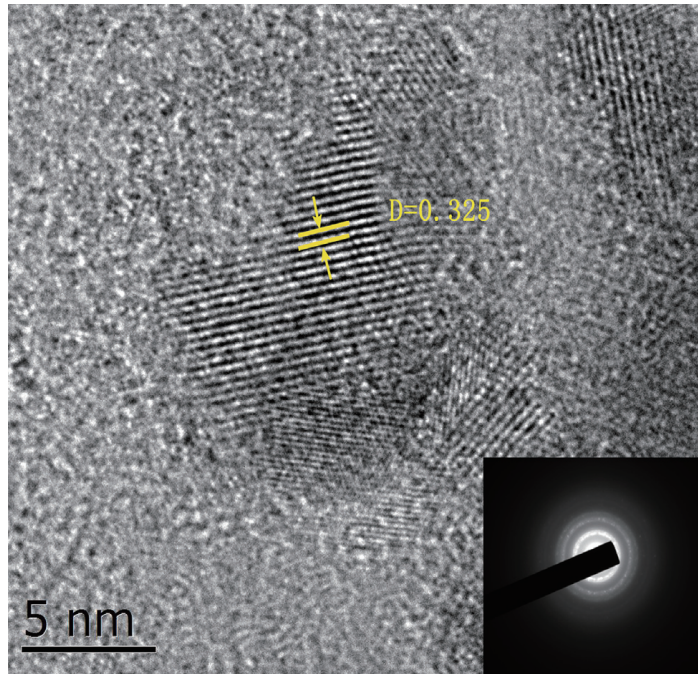

(b)

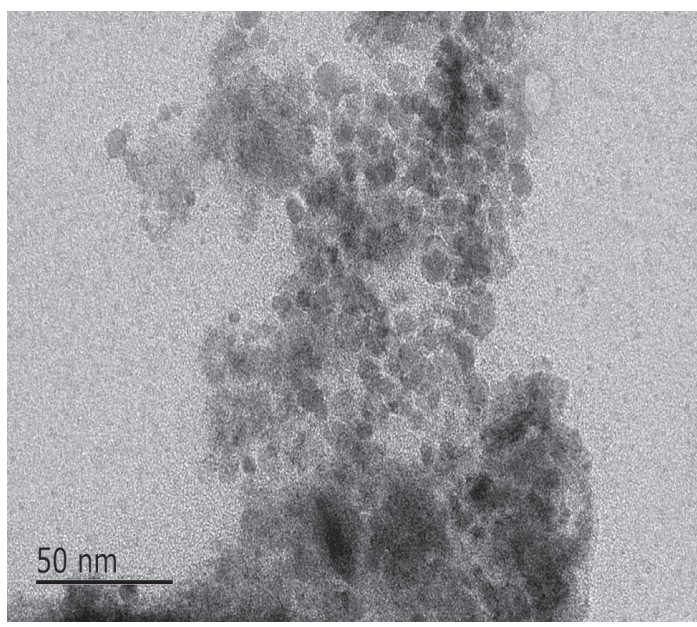

(d)

Figure 1: Characterization of as-prepared CuFeSe ${ }_{2}$ and MPEG-PCL@CuFeSe $e_{2}$ NPs. (a) TEM image of CuFeSe $e_{2}$. (b) HRTEM image with corresponding SAED pattern (inset). (c) The histogram for the measured particle size distribution. (d) TEM image of MPEG-PCL@CuFeSe 2 .

CT/MR imaging. As shown in Figure 4(a), from the CT images and Hounsfield unit (HU) values of different concentrations of MPEG-PCL@CuFeSe $e_{2}$ NPs in comparison with the clinically used iopromide (Omnipaque, General Electric Pharmaceutical Industry, Shanghai China), with the increasement of the concentration of MPEG-PCL@CuFeSe NPs and iopromide, the CT signal intensity was gradually enhanced, and at the same concentration, the images are brighter when MPEG-PCL@CuFeSe $e_{2}$ NPs are compared with the clinically used iopromide. In addition, the in vitro MRI imaging performance of MPEG-PCL@CuFeSe ${ }_{2} \mathrm{NPs}$ was evaluated with a clinically used 3.0 T MRI instrument. Figure 4(b) shows $T_{2}$-weighted images of MPEGPCL@CuFeSe $\mathrm{NPs}_{2}$. With the increasing concentration of MPEG-PCL@-CuFeSe $e_{2}$ NPs, the MR signal intensity was gradually decreasing, resulting in getting darker images.

3.4. In Vivo CT/MR Dual-Modality Imaging. Based on the in vitro dual-modal CT/MR contrast performance and good biocompatibility, we performed in vivo CT/MR imaging experiments of mPEG-PCL@CuFeSe $e_{2}$ nanoparticles in 


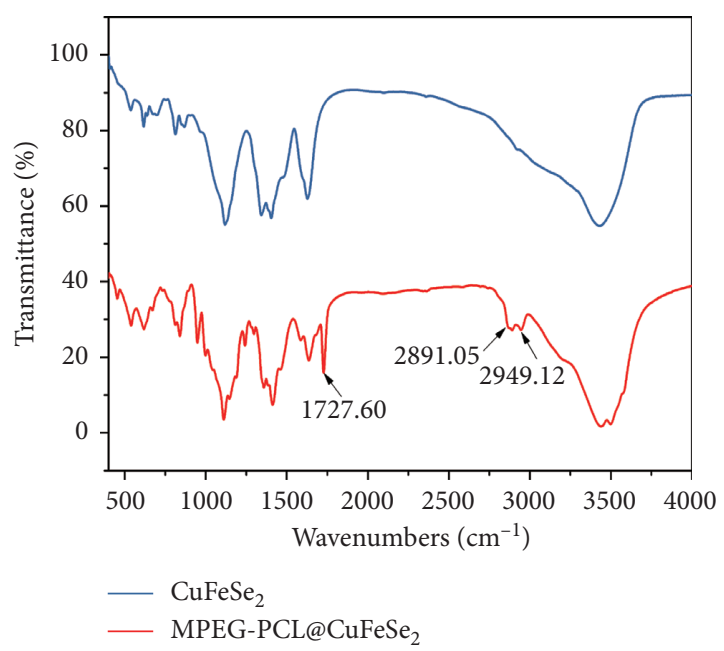

(a)

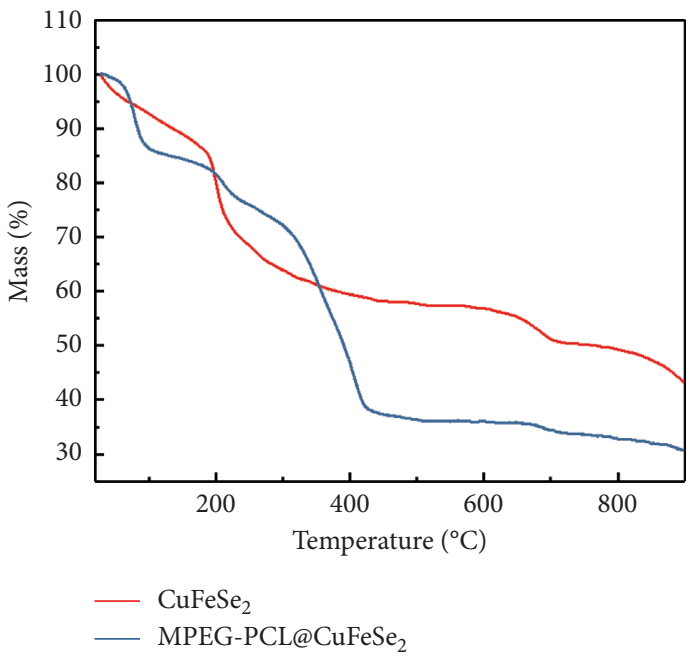

(c)

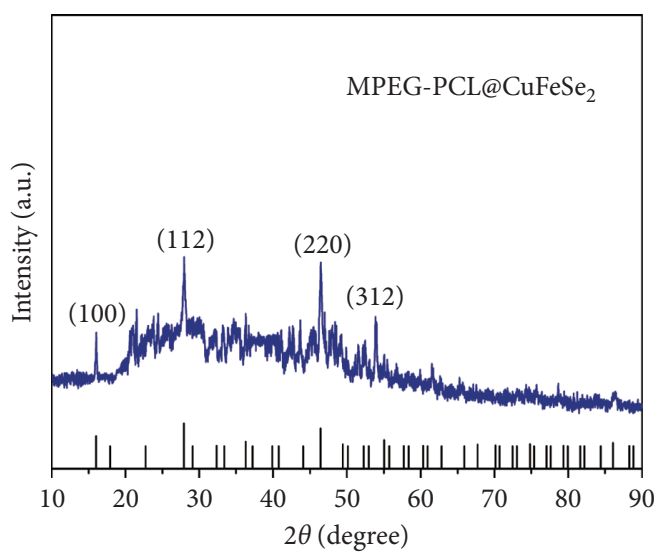

(b)

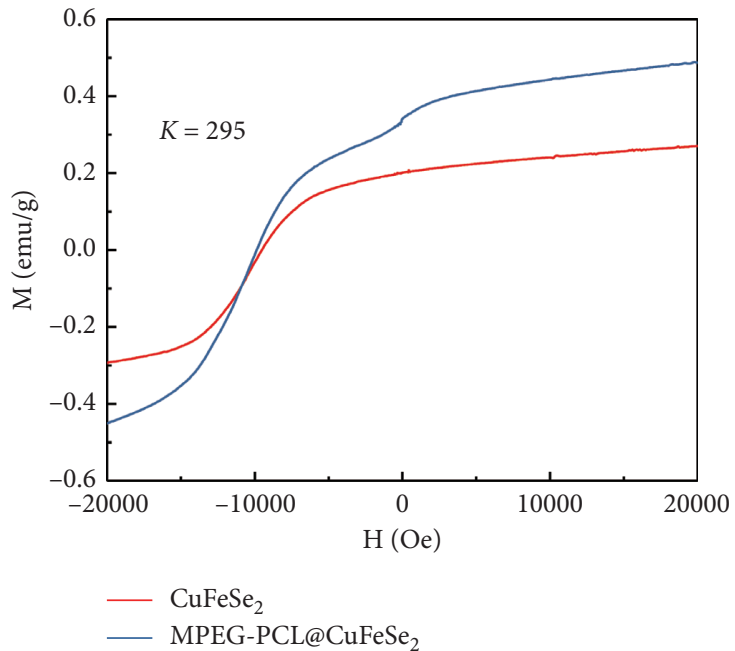

(d)

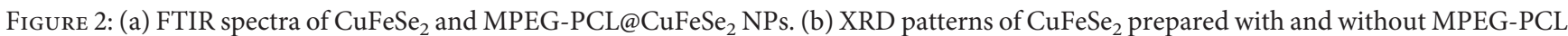
in comparison with the standard peaks of cubic berzelianite (JCPDS card no. 81-1959). (c) TGA curve of CuFeSe $e_{2}$ and MPEG-PCL@CuFeSe 2 NPs. (d) Magnetization plot of $\mathrm{CuFeSe}_{2}$ and MPEG-PCL@CuFeSe ${ }_{2}$ NPs as a function of the applied field at $295 \mathrm{~K}$.

TABLE 1: Hydrodynamic diameters and zeta potentials of NPs.

\begin{tabular}{lccr}
\hline Nanoparticles & Diameters $(\mathrm{d} / \mathrm{nm})$ & PDI & Zeta potential $(\varphi / \mathrm{mv})$ \\
\hline MPEG-PCL & $25.31 \pm 2.07$ & $0.263 \pm 0.527$ & $-21.03 \pm 1.53$ \\
MPEG-PCL@CuFeSe2 & $120.91 \pm 3.44$ & $0.183 \pm 0.007$ & $-10.85 \pm 2.59$ \\
\hline
\end{tabular}

PDI: polydispersity index.

transplanted mice. In the first place, CT images were acquired before and after intratumoral injection, and the tumor sites showed an enhancement with a higher CT value after administration of contrast agent when compared with those before injection (Figure 5(a)). Similar to CT imaging, $\mathrm{MR}$ images were acquired before and after intratumoral injection, and the tumor sites showed an enhancement with a lower $\mathrm{T}_{2} \mathrm{WI}$ MR signal intensity after administration of contrast agent when compared with those before injection (Figure 5(d)). The above results indicate the development of MPEG-PCL@CuFeSe ${ }_{2}$ has the potential to target bimodal $\mathrm{CT} / \mathrm{MR}$ imaging. 


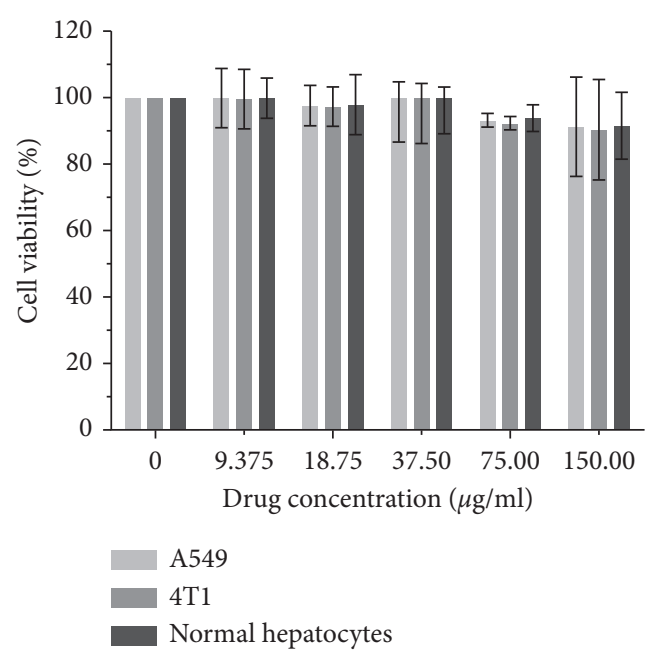

(a)

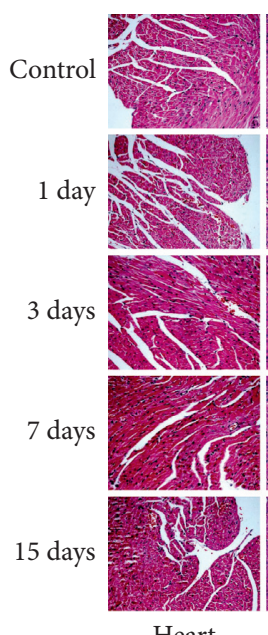

Heart

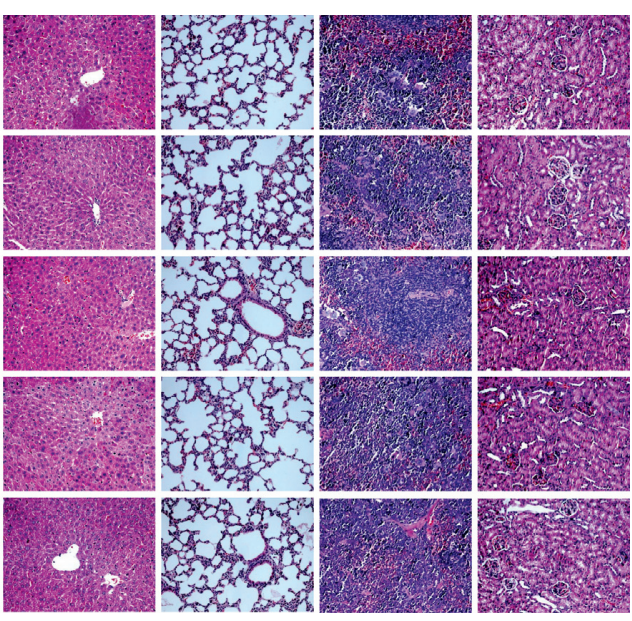

Lung

Spleen

(b)

Figure 3: (a) Cytotoxicity studies on 4T1, A549, and human normal liver cells after $24 \mathrm{~h}$ incubation with mPEG-PCL@CuFeSe 2 NPs at different concentrations. (b) Representative H\&E stained images of major organs including the heart, liver, spleen, lung, and kidney

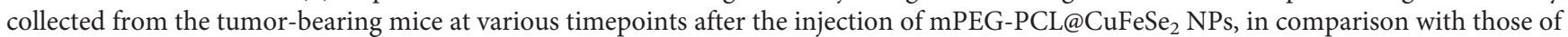
healthy mice. No obvious organ damage or lesions were observed after treatment.

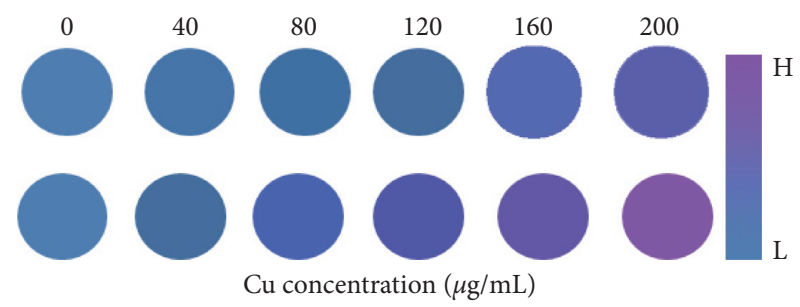

(a)

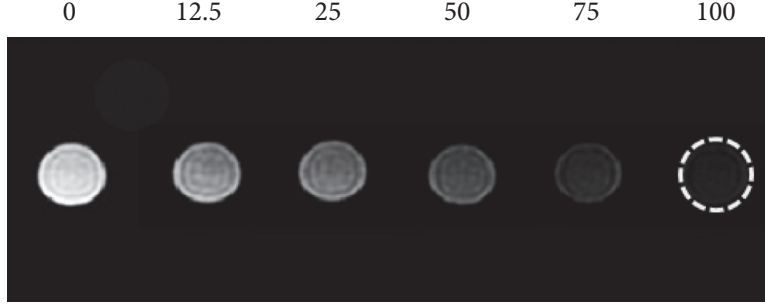

Fe concentration $(\mu \mathrm{g} / \mathrm{mL})$

(b)

Figure 4: (a) The CT images of mPEG-PCL@CuFeSe 2 NPs at different concentrations. (b) The $\mathrm{T}_{2}$-weighted MR images of mPEG-PCL@$\mathrm{CuFeSe}_{2}$ NPs at different Fe concentrations.

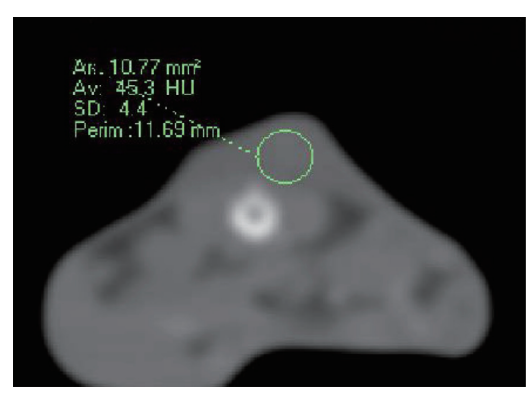

Pre

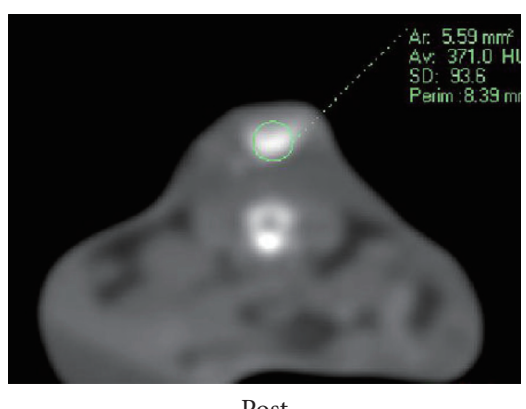

(a)

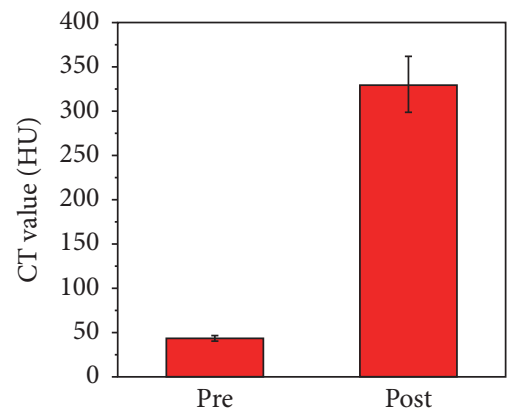

(b)

FIGURE 5: Continued. 


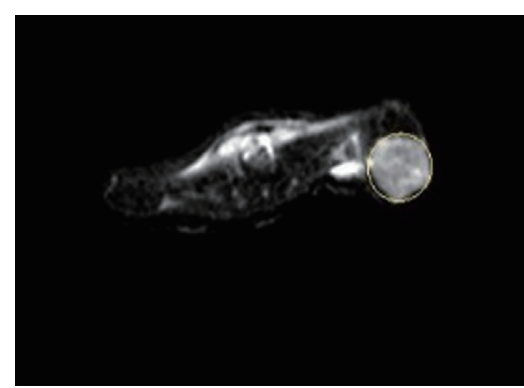

Pre

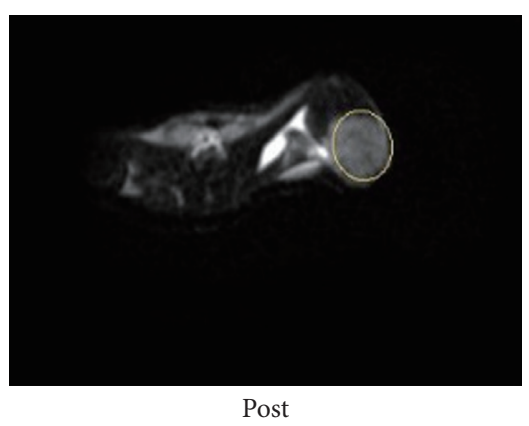

(c)

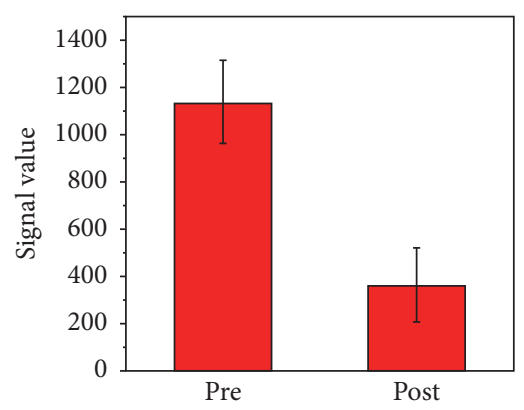

(d)

FIgURE 5: (a, b) CT images and CT values of mice collected pre- and post-intratumoral injection of mPEG-PCL@CuFeSe 2 NPs. (c, d) $\mathrm{T}_{2}$-weighted MR images and signal intensity of mice collected pre- and post-intratumoral injection of mPEG-PCL@CuFeSe ${ }_{2}$ NPs.

\section{Conclusions}

In summary, the MPEG-PCL copolymer-modified $\mathrm{CuFeSe}_{2}$ nanoparticles with favorable biological safety were successfully prepared by an environmentally friendly aqueous route under ambient conditions, and the MPEG-PCL@CuFeSe ${ }_{2}$ nanoparticles perform positive CT/MR contrast effect in vitro/in vivo. These excellent properties enable them to be a promising nanotheranostic agent for in vivo multimodal imaging.

\section{Data Availability}

The laboratory experimental data used to support the findings of this study are available from the first author upon request.

\section{Conflicts of Interest}

The authors declare that they have no conflicts of interest.

\section{Acknowledgments}

This study was supported from the Open Project of Key Laboratory of Nuclear Medicine and Molecular Imaging of Sichuan China (2018-12). The authors also are grateful for support from the Molecular Imaging Laboratory and Oncology Medical Laboratory of Southwest Medical University.

\section{References}

[1] L. Cheng, J. Liu, X. Gu et al., "PEGylated WS2Nanosheets as a multifunctional theranostic agent for in vivo dual-modal CT/ photoacoustic imaging guided photothermal therapy," Advanced Materials, vol. 26, no. 12, pp. 1886-1893, 2014.

[2] M. Zhou, R. Zhang, M. Huang et al., "A chelator-free multifunctional $[64 \mathrm{Cu}] \mathrm{CuS}$ nanoparticle platform for simultaneous micro-PET/CT imaging and photothermal ablation therapy," Journal of the American Chemical Society, vol. 132, no. 43, pp. 15351-15358, 2010.

[3] A. Dzik-Jurasz, C. Domenig, M. George et al., "Diffusion MRI for prediction of response of rectal cancer to chemoradiation," The Lancet, vol. 360, no. 9329, pp. 307-308, 2002.

[4] D.-M. Koh, E. Scurr, D. Collins et al., "Predicting response of colorectal hepatic metastasis: value of pretreatment apparent diffusion coefficients," American Journal of Roentgenology, vol. 188, no. 4, pp. 1001-1008, 2007.

[5] F. Jabeen, M. Najam-ul-Haq, R. Javeed, C. Huck, and G. Bonn, "Au-nanomaterials as a superior choice for near-infrared photothermal therapy," Molecules, vol. 19, no. 12, pp. 20580-20593, 2014.

[6] R. Ahmad, J. Fu, N. He, and S. Li, "Advanced gold nanomaterials for photothermal therapy of cancer," Journal of Nanoscience and Nanotechnology, vol. 4880, pp. 67-80, 2016.

[7] C. Cha, S. R. Shin, N. Annabi, M. R. Dokmeci, and A. Khademhosseini, "Carbon-based nanomaterials: multifunctional materials for biomedical engineering," ACS Nano, vol. 7, no. 4, pp. 2891-2897, 2013.

[8] Y. Shi, M. Liu, F. Deng et al., "Recent progress and development on polymeric nanomaterials for photothermal therapy: a brief overview," Journal of Materials Chemistry B, vol. 5, no. 2, 2016.

[9] K. Yang, L. Hu, X. Ma et al., "Multimodal imaging guided photothermal therapy using functionalized graphene nanosheets anchored with magnetic nanoparticles," Advanced Materials, vol. 24, no. 14, pp. 1868-1872, 2012.

[10] S. Wang, Q. Zhang, X. F. Luo et al., "Magnetic graphene-based nanotheranostic agent for dual-modality mapping guided photothermal therapy in regional lymph nodal metastasis of pancreatic cancer," Biomaterials, vol. 35, no. 35, pp. 9473-9483, 2014.

[11] S. Gao, L. Zhang, G. Wang et al., "Hybrid graphene/Au activatable theranostic agent for multimodalities imaging guided enhanced photothermal therapy," Biomaterials, vol. 79, no. 3, pp. 36-45, 2016.

[12] J. M. Delgado, G. D. de Delgado, M. Quintero, and J. C. Woolley, "The crystal structure of copper iron selenide, CuFeSe2," Materials Research Bulletin, vol. 27, no. 3, pp. 367-373, 1992.

[13] J. Lamazares, F. Gonzalez-Jimenez, E. Jaimes et al., "Magnetic, transport, X-ray diffraction and Mössbauer measurements on CuFeSe2," Journal of Magnetism and Magnetic Materials, vol. 104-107, no. 12, pp. 997-998, 1992.

[14] J. C. Bernede, N. Hamdadou, and A. Khelil, "X-ray photoelectron spectroscopy study of CuFeSe2 thin films," Journal of Electron Spectroscopy and Related Phenomena, vol. 141, no. 1, pp. 61-66, 2004.

[15] L. V. Kradinova, A. M. Polubotko, V. V. Popov, V. D. Prochukhan, Y. V. Rud, and V. E. Skoriukin, "Novel zero-gap compounds, magnetics: CuFeS2and CuFeTe2," 
Semiconductor Science and Technology, vol. 8, no. 8, p. 1616, 1993.

[16] Y.-K. Hsu, Y.-G. Lin, and Y.-C. Chen, "One-pot synthesis of CuFeSe2 cuboid nanoparticles," Materials Research Bulletin, vol. 46, no. 11, pp. 2117-2119, 2011.

[17] X. Jiang, S. Zhang, F. Ren et al., "Ultrasmall magnetic CuFeSe2 ternary nanocrystals for multimodal imaging guided photothermal therapy of cancer," ACS Nano, vol. 11, no. 6, pp. 5633-5645, 2017.

[18] W. Dang, T. Li, B. Li et al., "A bifunctional scaffold with $\mathrm{CuFeSe} 2$ nanocrystals for tumor therapy and bone reconstruction," Biomaterials, vol. 160, pp. 92-106, 2018.

[19] Q. Lu, J. Hu, K. Tang, B. Deng, Y. Qian, and Y. Li, "The synthesis of $\mathrm{CuFeSe} 2$ through a solventothermal process," Journal of Crystal Growth, vol. 217, no. 3, pp. 271-273, 2000.

[20] M. S. Kim, H. Hyun, K. S. Seo et al., "Preparation and characterization of MPEG-PCL diblock copolymers with thermo-responsive sol-gel-sol phase transition," Journal of Polymer Science Part A Polymer Chemistry, vol. 44, no. 18, pp. 5413-5423, 2006.

[21] B. L. Wang, Y. M. Shen, Q. W. Zhang et al., "Codelivery of curcumin and doxorubicin by MPEG-PCL results in improved efficacy of systemically administered chemotherapy in mice with lung cancer," International Journal of Nanomedicine, vol. 8, no. 1, p. 3521, 2013.

[22] H. Kheiri Manjili, P. Ghasemi, H. Malvandi, M. S. Mousavi, E. Attari, and H. Danafar, "Pharmacokinetics and in vivo delivery of curcumin by copolymeric mPEG-PCL micelles," European Journal of Pharmaceutics and Biopharmaceutics, vol. 116, pp. 17-30, 2017.

[23] E. Blanco, C. W. Kessinger, B. D. Sumer, and J. Gao, "Multifunctional micellar nanomedicine for cancer therapy," Experimental Biology and Medicine, vol. 234, no. 2, pp. 123-131, 2009.

[24] R. Weissleder and U. Mahmood, "Molecular imaging," $R a-$ diology, vol. 219, no. 2, pp. 316-333, 2001.

[25] D.-E. Lee, H. Koo, I.-C. Sun, J. H. Ryu, K. Kim, and I. C. Kwon, "Multifunctional nanoparticles for multimodal imaging and theragnosis," Chemical Society Reviews, vol. 41, no. 7, pp. 2656-2672, 2012.

[26] P. Padmanabhan, A. Kumar, S. Kumar, R. K. Chaudhary, and B. Gulyás, "Nanoparticles in practice for molecular-imaging applications: an overview," Acta Biomaterialia, vol. 41, pp. 1-16, 2016.

[27] L. Chen, X. Ni, H. Zhang et al., "Preparation, characterization, in vitro and in vivo anti-tumor effect of thalidomide nanoparticles on lung cancer," International Journal of Nanomedicine, vol. 13, pp. 2463-2476, 2018. 